\title{
„Im Westen viel Neues“ - Jahrestagung der NOUV 2017 zog von Hamburg nach Dortmund und das Konzept ging auf
}

Im Westen gibt's Norden - das war das Motto der Jahrestagung der NOUV 2017. Die Tagungspräsidenten PD Dr. Jörg Franke, Prof. Dr. Christian Lüring und Prof. Dr. Thomas A. Schildhauer freuen sich, dass ihr Konzept aufgegangen ist: Über 750 registrierte Teilnehmer haben sich von Donnerstagfrüh bis Samstagvormittag in der Westfalenhalle in Dortmund getroffen, Vorträge gehalten, diskutiert und sich ausgetauscht. Warum überhaupt Dortmund? Den meisten wird die Jahrestagung der NOUV in Hamburg ein Begriff sein. Da das dortige Kongresszentrum aber in 2017 renoviert wird, musste ein neuer Kongressort gefunden werden. Der Vorstand der NOUV entschied sich, eine Tradition zu erhalten, indem er mit einer Tradition brach: den Kongress fortführen, aber den Ort wechseln. Insgesamt ein traditionsbewusster Schritt, geht doch die Norddeutsche Orthopäden- und Unfallchirurgenvereinigung auf die Vereinigung nordwestdeutscher Chirurgen zurück. Das Kongresskonzept sollte behutsam modernisiert werden. Folgerichtig wurde eine hohe Bandbreite der Themen angeboten: ein eigenständiges Fortbildungsprogramm für D-Ärzte, ein AE Forum, ein $A E$ Basis-Kurs Hüft- und Kniegelenk, separate Angebote für Physiotherapeuten sowie interessante Themen für noch in der Ausbildung befindliche Assistenz- und Fachärzte. Die wissenschaftlichen Schwerpunkte des Kongressprogramms der NOUV-Jahrestagung 2017 waren individuelle Endopro- thetik, Knorpelreparation, Kinderorthopädie, Medizin in Grenzsituationen, Alterstrauma, posttraumatische Fehlstellungen, Gelenkinstabilitäten akut und chronisch, kindliche Frakturen, implantatassoziierte Infekte, Deformitäten der Wirbelsäule des Erwachsenen und minimalinvasive Chirurgie an der Wirbelsäule. Neben den Abstracteinreichungen wurden die Themen auch durch die unterschiedlichsten Fachgesellschaften unterstützt, so gab es eigene Sitzungen der AGA, der DVSE, der Vereinigung für Kinderorthopädie, von Ärzte ohne Grenzen, der AGITEC, der Vereinigung Technische Orthopädie und der D.A.F.

Dieses Spektrum wurde vom Fachpublikum sehr gut angenommen: die Sitzungen waren durchweg gut gefüllt und die Stimmung unter den Teilnehmern war sehr positiv. Insbesondere der Standortwechsel wurde als gelungen bewertet: das Kongresszentrum in der Dortmunder Westfalenhalle war räumlich gut an die Größe des Kongresses angepasst, die Industrieausstellung als Zentrum wurde in den Pausen ausgiebig genutzt und bot viel Gelegenheit zur Diskussion zwischen Fachkollegen und Industrievertretern. Die Säle waren gut erreichbar und die Wege kurz, ganz wie es sich für einen Kongress dieser Größe gehört. Man begegnete sich und hatte ausreichend Gelegenheit, zu netzwerken und miteinander in Kontakt zu kommen.
Wissenschaftliche Highlights waren die Verleihung des Albert-Hoffa-Preises an Lukas Weiser aus Göttingen für seine Arbeit „Cement augmentation versus extended dorsal instrumentation in the treatment of osteoporotic vertebral fractures“. Darüber hinaus wurden 3 Posterpreise vergeben, die mit jeweils $500 €$ dotiert waren. Traditionell wird auch der AXIS-Forschungspreis auf dem NOUV vergeben. Dieser von der Fa. Implantcast gestiftete Preis wurde in diesem Jahr auf 2 Kandidaten aufgeteilt: Pia Reimann aus Dortmund erhielt den Preis für ihre Arbeit mit dem Titel „Individuelle vs. Konventionelle Knieendoprothetik - Unterscheiden sich die Frühergebnisse?“. Daniel Körner aus Tübingen erhielt den Preis für die Arbeit „Parameters influencing complaints and joint function in patients with osteochondral lesions of the ankle - an investigation based on data from the German Cartilage Registry (KnorpelRegister DGOU)“.

Insgesamt gesehen war der Schritt, den Kongress von Hamburg nach Dortmund zu verlegen, mutig, aber richtig und es zeigt sich, dass das Konzept aufgegangen ist.

\section{Korrespondenzadresse}

Prof. Dr. med. Christian Lüring

Orthopädische Klinik

Klinikum Dortmund

christian.luering@klinikumdo.de 